\title{
Modeling scenarios of earthquake-generated tsunamis for Vietnam coasts
}

\author{
Davide Bisignano \\ Università degli Studi di Trieste, Department of Geosciences \\ Via E. Weiss 4, Trieste, Italy \\ E-mail:_davide.bisignano@gmail.com
}

\section{Fabio Romanelli}

Universita' degli studi di Trieste, Department of Geosciences

Via E. Weiss 4, Trieste, Italy

E-mail: romanel@units.it

\section{Antonella Peresan ${ }^{1}$}

The Abdus Salam International Centre for Theoretical Physics

ICTP SAND Group

Strada Costiera 11, Trieste. Italy

E-mail:peresan@ictp.it

\begin{abstract}
Modeling a hazard scenario has the main purpose to assess the maximum threat expected from a studied phenomenon in a certain area and to give specific directives to the local authorities in order to prevent and mitigate serious consequences on the population, the infrastructures and the environment. To build scenario-based tsunami hazard maps for a specific coastal area one has first to characterize the seismic sources (or other tsunamigenic events, not considered in this study) and select the earthquake scenarios that can drive the hazard. By means of the modeling we then calculate the maximum amplitude of the vertical displacement of the water particles on the sea surface and the travel time of the maximum amplitude peak, since they are the most relevant aspects of the tsunami wave and also are the only characteristics always recorded in the chronicles and therefore in catalogues. In this work we have used this approach to compute earthquake generated tsunami hazard along the Vietnam coasts. The results of our calculations suggest that a tsunami with maximum amplitude up to ten meters can be expected also in the South China Sea, in agreement with a number of historical events reported in the catalogues. Considering the low level of monitoring of the South China Sea and the advanced anthropic setting of the Vietnam coasts, and their high level of vulnerability, we hope that the results can be used as a preliminary knowledge basis to: design early warning systems, reduce tsunami risk and plan land-use for the Vietnam coasts.
\end{abstract}

\footnotetext{
${ }^{1}$ Speaker
} 
The International Symposium on Grids and Clouds and the Open Grid Forum Academia Sinica, Taipei, Taiwan

March 19 - 25, 2011 


\section{Introduction}

Tsunami phenomenon, that has reached a global attention with the 2004's event in Sumatra, has strongly come back into actuality in these days because of the recent devastating earthquake in Japan (March 11th, M 9.0) where more than 20000 people were killed and whose damages at nuclear plants of Fukushima are still on the chronicles in these days. These and other catastrophes call for an increased attention in dealing with tsunami disasters, both on alert systems (e.g. DART systems) and hazard maps. In particular the unexpected magnitude of the latter event and consequent inefficiency, in particular for tsunami barriers, impose us to put new consideration on the concept of "maximum credible earthquake" and on hazard-scenarios based on it.

Modeling a hazard scenario has the main purpose to assess the maximum threat expected from a studied phenomenon in a certain area and to give specific directives to the local authorities in order to prevent and mitigate serious consequences on the population, the infrastructures and the environment. To build scenario-based tsunami hazard maps for a specific coastal area one has first to characterize the seismic sources (or other tsunamigenic events, not considered in this study) and select the earthquake scenarios that can drive the hazard. By means of the modeling we then calculate the maximum amplitude of the vertical displacement of the water particles on the sea surface and the travel time of the maximum amplitude peak, since they are the most relevant aspects of the tsunami wave and also are the only characteristics always recorded in the chronicles and therefore in catalogues. The horizontal displacement field is calculated too, and, on average, it exceeds the vertical one by an order of magnitude approximately (this accounts for the great inundating power of tsunami waves with respect to wind driven ones). It is important to point out that the extremely efficient analytical modeling techniques (computation times are of the order of seconds and are bound to decrease with the natural rate of improvement of computers) for real time simulations can be very useful also for integration in a Tsunami Warning System, since they can be compared with real time incoming open-sea level data, in order to validate, or close, an impending alarm. This work is a development of Bisignano and Romanelli (2009), with the main difference of the inclusion of a source along Manila Trench whose maximum expected magnitude, following other authors, has been considered $M=9$; the importance of this study is given by two factors: a) the low level of monitoring of the South China Sea; b) the advanced anthropic setting of the Vietnam coasts and their high level of vulnerability.

\section{Tsunami Modeling}

The traditional approach to model tsunami generation is based on solving the hydrodynamic equations with boundary conditions at the ocean floor corresponding to a static displacement caused by the earthquake source. Another well developed approach is based on the modal theory (e.g. Ward, 1980; Comer, 1984). The former approach assumes that the seabottom is rigid and, after its initial displacement, it does not interact with the fluid: they are partially coupled. According to the modal theory the ocean and solid Earth are fully coupled: with this dynamic condition the excitation of the seismic source can be naturally included. 


\subsection{The modal method}

The approach we make use here for modeling tsunamis generated by offshore earthquakes is the extension, performed by Panza et al. (2000) to the case of tsunami propagation, of the well-known modal theory and therefore we simply refer to it as "modal method". In this approach it is assumed that the ocean and the solid Earth are fully coupled.

From the mathematical point of view in the modal approach the equations of motion are solved for a multi-layered model structure, so the set of equations is converted into a matrix problem in which to look for eigenvalues and eigenfunctions (e.g. Haskell, 1953; Knopoff, 1964). In general, the modal theory gives a solution corresponding to the exact boundary conditions, and so it is easily extended to models with slightly varying thickness of the water layer. Therefore, the modal method allows us to calculate synthetic signals for both laterally homogeneous (1D) and laterally heterogeneous (2D) structures. For the 2D case, the structural model is parameterized by a number of 1D structures put in series along the profile from the source to the receiving site. Every liquid layer is considered to be homogeneous and incompressible, no vertical stratification of the water is considered.

The parameterization of the bathymetry could be important for the longer source-site paths, since it can significantly influence travel times. However, in this study, in order to efficiently compute a large number of synthetic signals, we adopted a simplified description of 2D media; specifically, for every source-receiver path, we used just two different structures: one at the source and one at the site. Our aim is to provide quick indications about the level of the wave we can expect from different possible earthquake scenarios, rather than to compute detailed inundation maps. So, at this first order level, the fast calculation of a very large number of scenarios, is more relevant than a very high level of precision. Due to the use of simplified models, the mareograms are computed only along straight segments from the source to the receiver sites, neglecting all three-dimensional effects, such as refraction and diffraction. When analysing the results it is necessary to consider that the applied model is linear, whereas topographic and bathymetric effects on the shore are not; therefore the results have to be considered as open sea level (i.e. in a range from deep ocean to about $50 \mathrm{~m}$ depth), which can be used as boundary conditions for detailed inundation models to compute run-up and inundation maps.

\subsection{The extended source model}

The point-source approximation holds for source-receiver distances greater than those of the order of the fault length; this assumption is not always valid and a modeling of an extended source is requested.

In this work to obtain mareograms for the extended source we have developed a code that uses the data of the slip distribution along the fault obtained by a stochastic procedure using another program (PULSYN) developed at the ICTP by Prof. A. Gusev (Gusev and Pavlov, 2006). This last program discretizes the fault and assigns a value of the slip and of rupture time to each sub-source (see Figure 1). The characteristic of each sub-source is then used as an independent source to model the tsunami and the sum of all the signals obtained gives us a final mareogram for the extended source. 


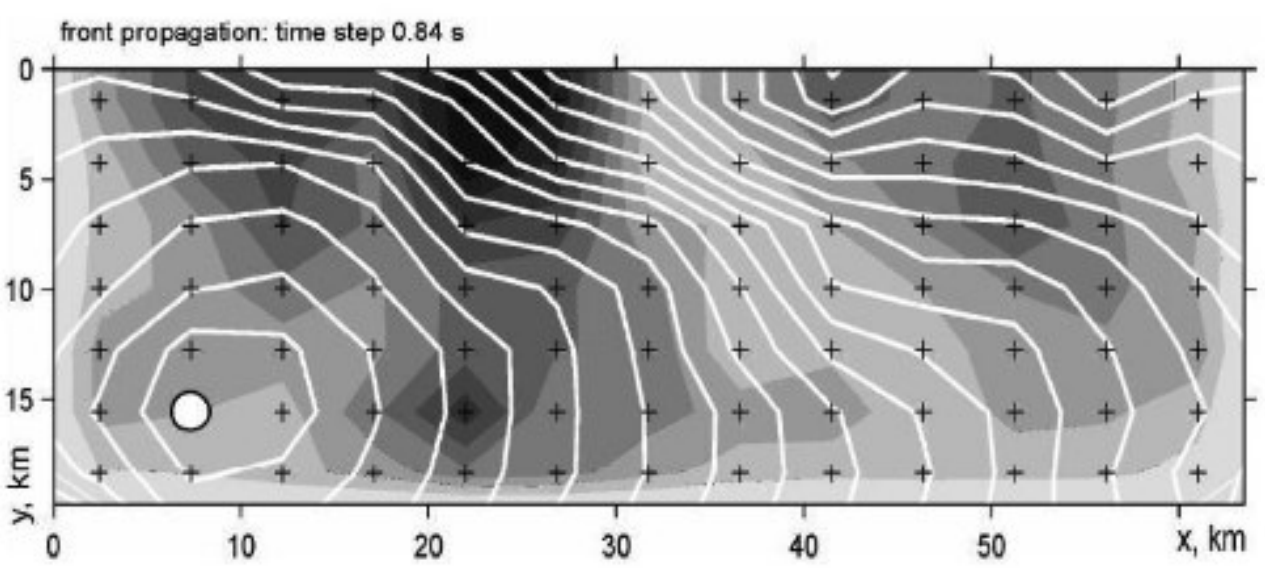

Figure 1: An example of 2D final slip and rupture history obtained with Pulsyn (Gusev et. al 2006)

\subsection{Test on $11^{\text {th }}$ March 2011 Tohoku earthquake}

To show the validity of these methodologies we have performed some test on the $11^{\text {th }}$ March event in Japan. In particular we have used the finite source model by Gavin Hayes from USGS (http://earthquake.usgs.gov/earthquakes/eqinthenews/2011/usc0001xgp/finite_fault.php) and, using a simplified 2D propagation model, we have computed signals at 3 DART buoys, in particular: buoy 21418 , buoy 21401 , buoy 21413 . The mutual position of source and buoys are shown in figure 2; the comparison between modeled tsunamigrams and real data is provided in figures $3,4,5$. The agreement with recordings is fairly good, particularly considering the very fast computation time (in the order of few seconds, once the parametric files with sources and bathymetry are defined). Further tests can be performed, anyway, in order to optimize the bathymetry parameterization for the path. For more complex bathymetries, with strong lateral and radial gradients, our method suffers for its proper limitation. The possibility to perform, at very low computational cost, a large number of parametric tests, accounting for different possible configurations of finite sources, could be very important when computing hazard maps and to characterize the related uncertainties.

\section{Earthquake induced tsunami scenarios}

To calculate tsunami hazard scenarios we first investigate the available historical data from the earthquake catalogues. Since for some areas the available earthquake catalogues could be incomplete or contain errors, the choice of earthquake scenarios has to include also the geological and seismotectonic information: this leads to a definition of a set of selected tsunamigenic zones with a distribution of focal mechanisms. Once that the earthquake scenarios and the structural models are chosen, the modal method is used to compute the synthetic tsunamigrams for the selected receiver sites or, for a gridded zone, to compute hazard maps. These maps report the maximum heights, computed in the linear regime, and their arrival times. The efficiency of the modal method permits also to map the tsunami propagation in extended basins for many earthquake scenarios. This would permit to obtain easily a wide data-base of pre-computed signals that can be used with an offshore warning system (e.g. Dart System) to be compared with real-time data. 


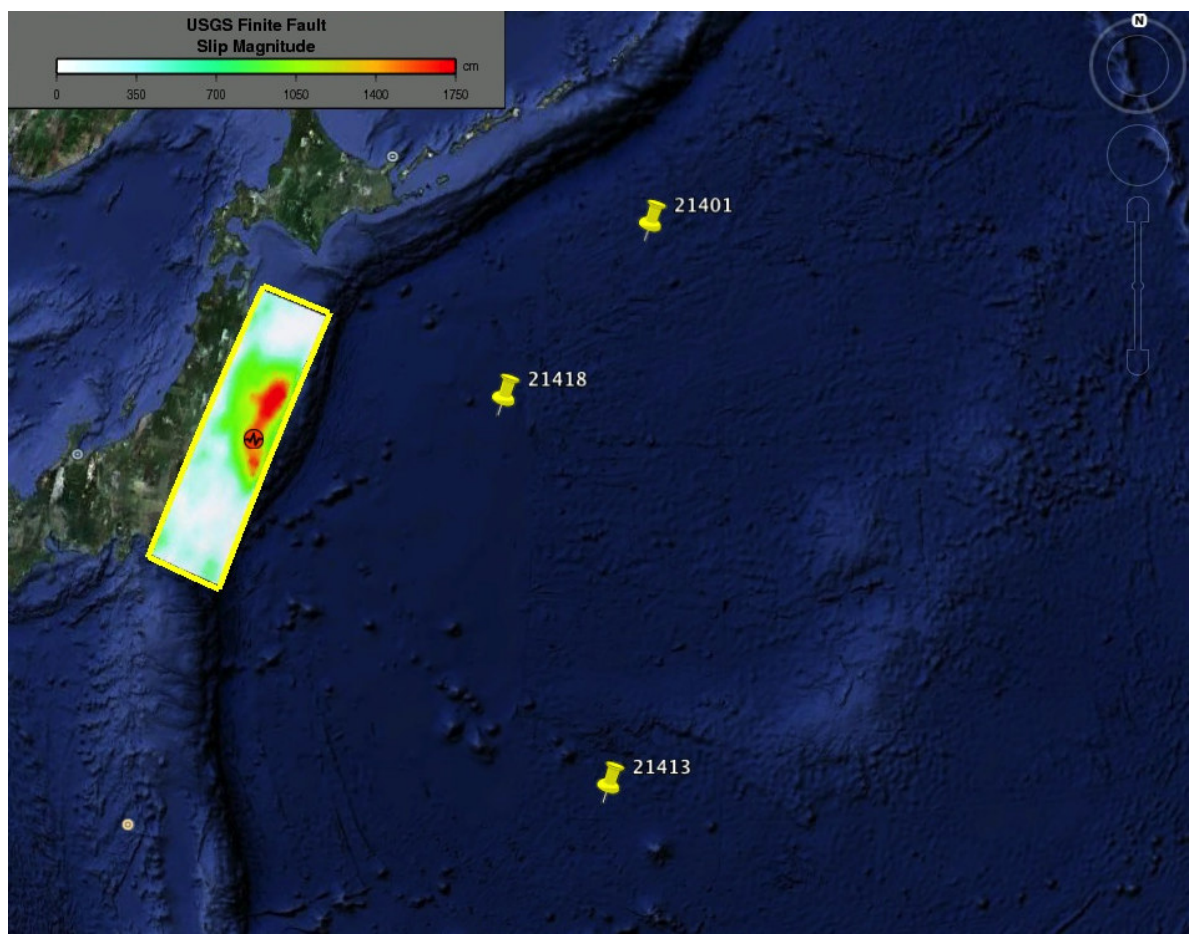

Figure 2. Source model and DART buoys

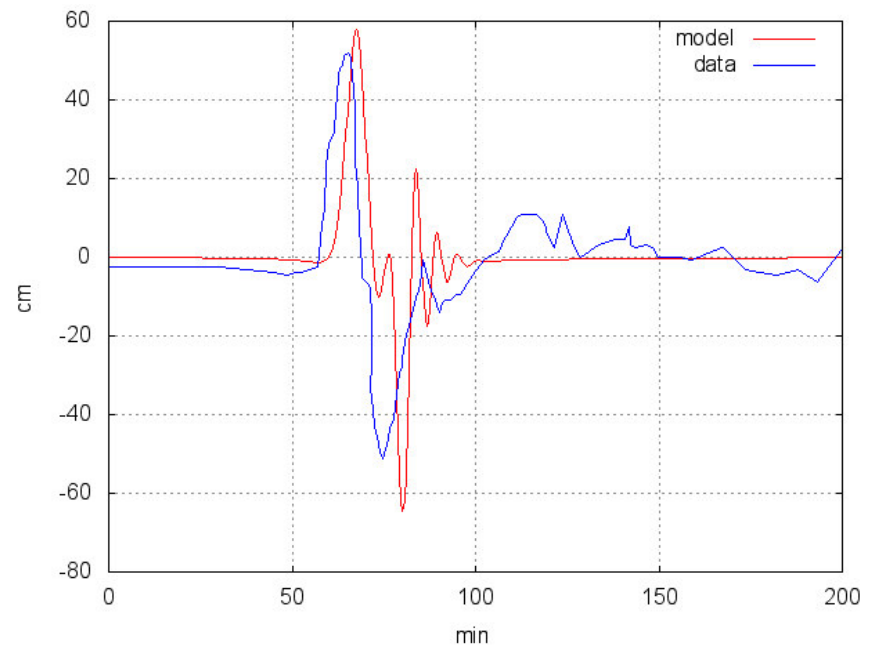

Figure 3. DART buoy 21401 


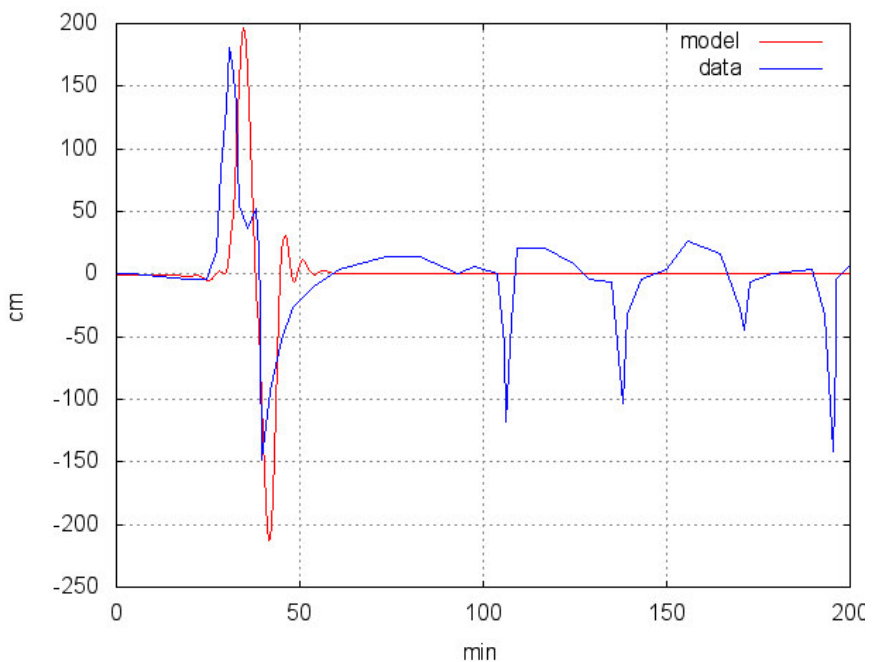

Figure 4. DART buoy 21418

Figure 5. DART buoy 21413

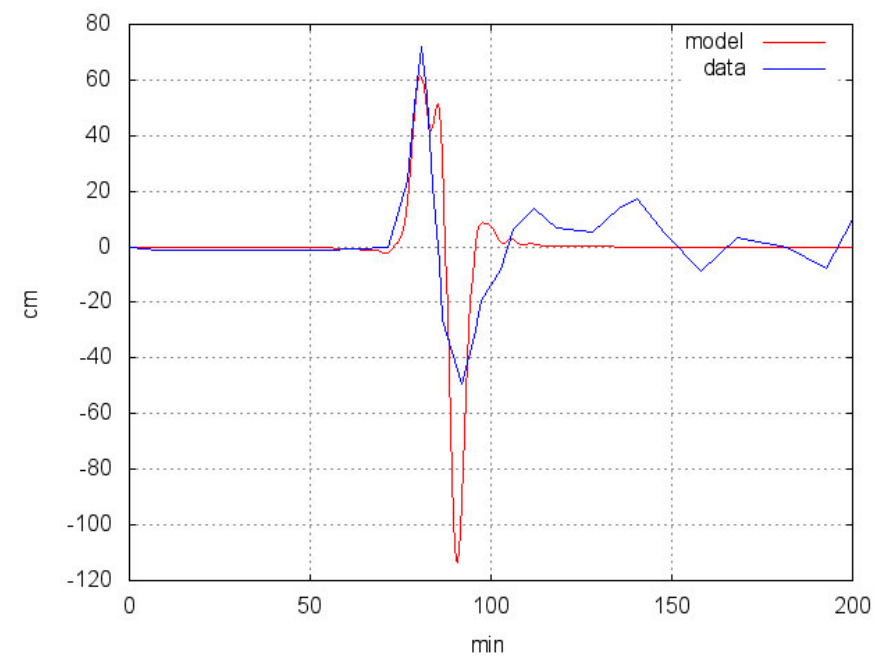

\subsection{Hazard along the Vietnam coast}

This study is a development of the work "Earthquake generated tsunami hazard scenarios along Vietnam coast (Bisignano and Romanelli 2009); the main improvement concerns the inclusion of a new earthquake source in the central part of Manila Trench, source 7 in figure 6.

Following for example Liu et al. (2007) and having in mind the high level of seismic activity this structure, we have decided to include it in our analysis, and in fact this turns out to be the most effective among the tsunami sources that may affect Vietnam coasts. Finally we have computed historical and maximum credible scenarios: the first is calculated from maximum magnitude earthquakes as reported in the last 100 years, while in the second we have assigned a maximum credible event for every source, referring not only to information from the statistical analysis of earthquake catalogues, but also to any available information about the seismogenic potential of fault systems. 


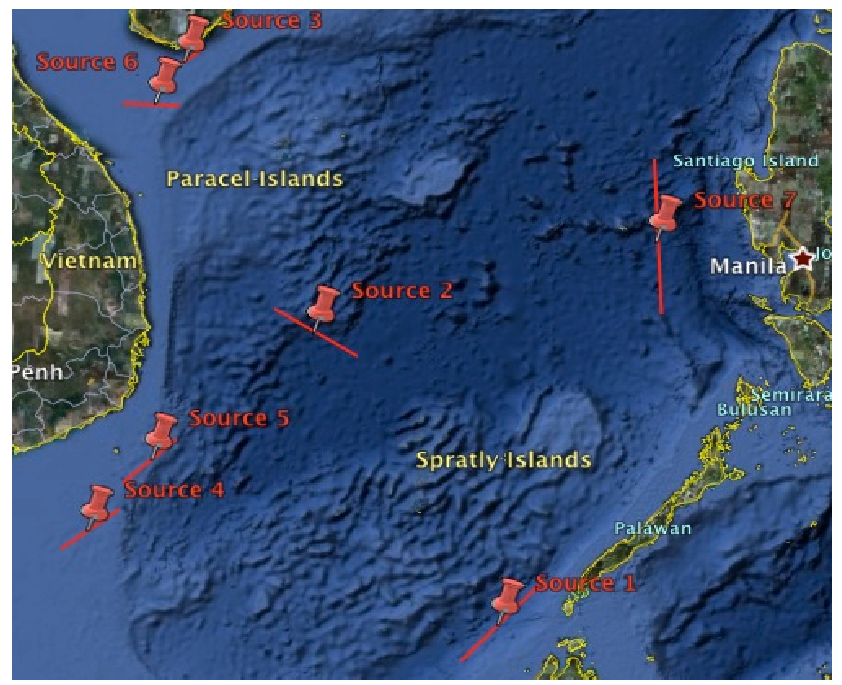

Figure 6. Map of selected sources (red segments with pins)

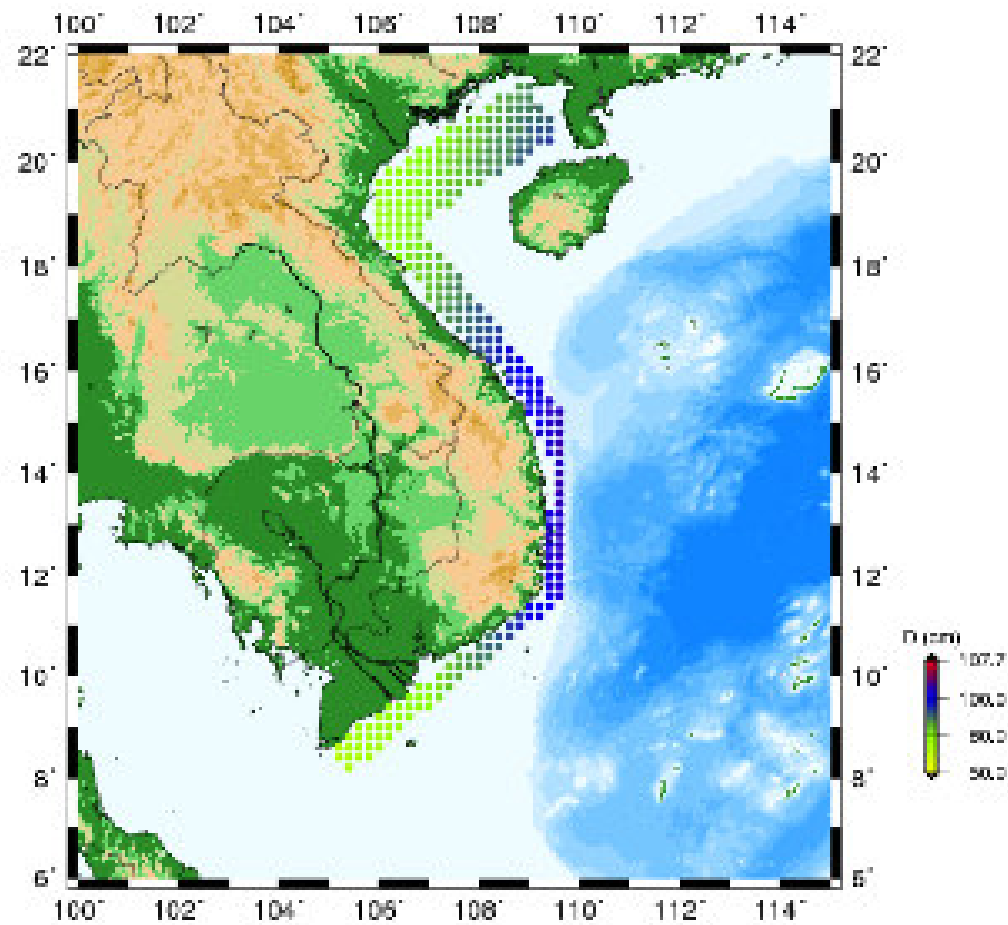

Figure 7. Aggregate hazard scenario from maximum historical earthquakes for the selected sources shown in figure 6.

The obtained results are shown in figures 7 and 8; in both cases it is evident the main contribution provided by the source in Manila Trench. In particular, the results from maximum credible scenario (where we have assigned $M=9$ ), with about 10 meters wave height on the central part of Vietnam coast, are in accordance with simulations of Ca and Xuyen (2008) and with paleotsunamis traces.

The values of historical hazard scenario are in agreement with historical chronicles, which do not report records of important tsunami related to seismic sources in Vietnam in the recent past. 
This obviously does not indicate that Vietnam coasts can be considered safe from tsunami, because of the possibility of great earthquakes $(M \geq 8)$ in Manila Trench, as well as due to the insufficient knowledge about sources in the western and central part of South China Sea. Moreover there are also reports of tsunamis not directly linked to earthquakes, suggesting that also other factors like volcanoes or submarine landslides should be considered.

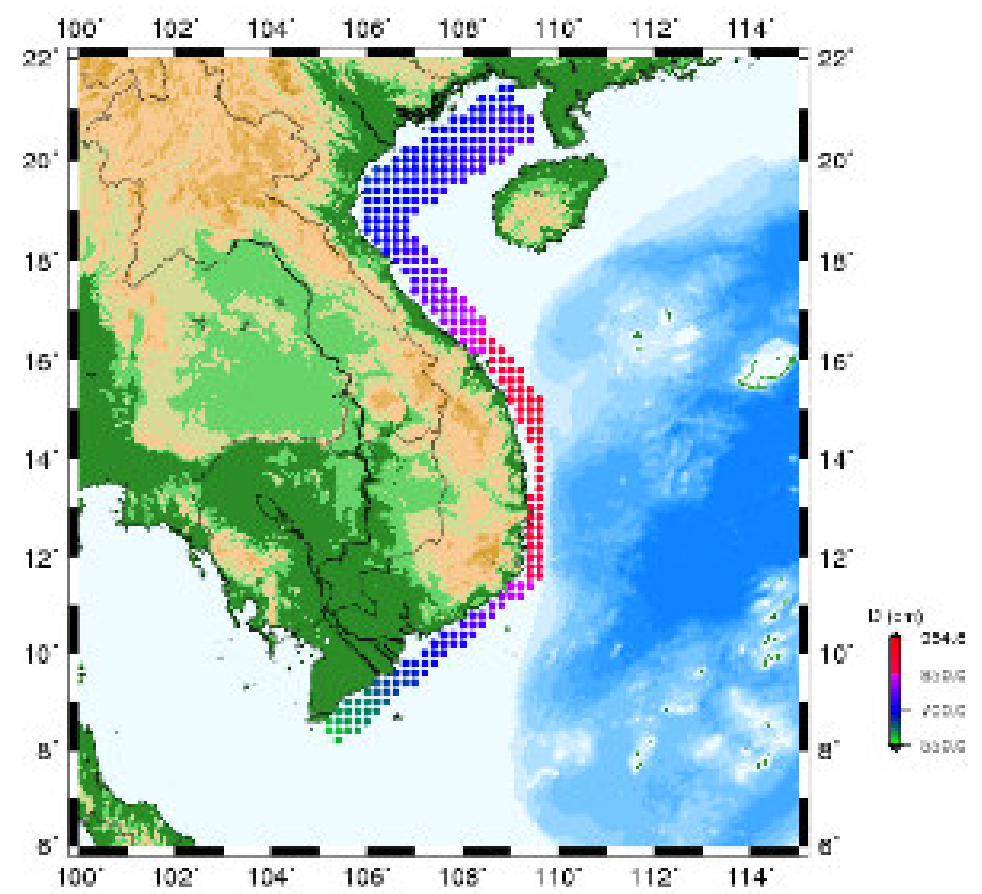

Figure 8. Aggregate hazard scenario from maximum credible earthquakes for selected sources shown in figure 6.

\section{Conclusions}

The considered method, based on modal technique, allowed us to define a set of earthquake-generated tsunamis scenarios for the Vietnam coast, using the current knowledge of the physical process of earthquake generation and wave propagation and the literature about seismicity in South China Sea zone. The results of our modeling suggest that a tsunami with maximum amplitude up to ten meters can be expected also in the South China Sea, in agreement with evidences of reported paleo-tsunami. The main hazard seems related to the source in Manila Trench, for which we have used a maximum expected value of magnitude $M=9$; however the proximity to the coast of other, less studied, potential sources would impose further studies also on those faults zones.

Considering the low level of monitoring of the South China Sea and the advanced anthropic setting of the Vietnam coasts, and their high level of vulnerability, we hope that the results can be used as a preliminary knowledge basis to: design early warning systems, reduce tsunami risk and plan land-use for the Vietnam coasts.

For what concerns the method we have used we can say that in situation with quite simple bathymetry it has shown a good accordance with measured data. Moreover its very low 
computational cost would permit to compute a lot of scenarios, especially permitting a lot of parametric tests on different finite source possible configurations, and these features could be useful both for hazard assessment and for pre-computing a lot of scenarios in the aim of realtime tsunami warning.

\section{Acknowledgments}

This work has greatly benefited from stimulating discussions with Giuliano Panza, Cao Dinh Trieu, as well as from the precious collaboration of many colleagues from the Institute of Geophysics, Vietnam Academy of Science and Technology. The research has been supported by the MAE-MOST Italian-Vietnamese bilateral Executive Programme in Science and Technology for the years 2006-2008 and 2009-2011.

\section{References}

[1] Bisignano D., Romanelli F., Earthquake generated tsunami hazard scenarios along the Vietnam coasts, in proceedings of IAEA International Workshop on External Flooding Hazard at Nuclear Plant Sites in Commemoration of the 5 years of the 2004 Indian Ocean Tsunami, Kalpakkam (India), 11-15 January 2010

[2] Cao Dinh Trieu, $Q$ Structure and stress pattern of the Earth Crust of Vietnam territory. Contribution of the Institute of Geophysics from 1987 to 1997, Hanoi.

[3] R. P. Comer, The tsunami mode of a flat earth and its excitation by earthquake sources, Geophys. J. R. Astr. Soc., 77,1-28. 1984

[4] Gusev A.A. and Pavlov V., Wideband simulation of earthquake ground motion by a spectrummatching, multiple-pulse technique, in proceedings, First European Conference on Earthquake Engineering and Seismology. (a joint event of the 13th ECEE \& 30th Generale Assembly of the ESC). 2006.

[5] Haskell, N.A., The dispersion of surface waves in multilayered media. Ge Bull. Seism. Soc. Am., 17, 34. 1953.

[6] Kirby S., Geist E., Lee W.H.K., School D. and Blakely R., Tsunami Source Characterization for Western Pacific Subduction Zones: A Preliminary Report, A Preliminary Report in "DART Network Optimization: 2005 Workshop Report”: NOAA Techinal Memorandum ERL PML.

[7] Knopoff L., A matrix method for elastic wave problems. Bull. Seism. Soc. Am., 431-438, 1964.

[8] Liu Y., Santos D., Wang S.M., Shi Y., Liu H., Yuen D. A., Tsunami Hazard along Chinese coast from potential earthquakes in South China Sea. Physics of The Earth and Planetary Interiors, Vol 163, Is 1-4, Pag 233-244. 2007.

[9] Panza G.F., Romanelli F. and Yanovskaya, T., Synthetic Tsunami mareograms for realistic oceanic models. Geophysical Journal International, 141, 498-508, 2000.

[10] Vu Than Ca \& Nguyen Dinh Xuyen., Tsunami risk along Vietnamise coast. Journal of Water Resources an Enviromental Engineering, 23, 24-33. 2008

[11] Ward S. N., Relationship of tsunami generation and earthquake source, J. Phys. Earth, 28 , 441474. 1980. 